\title{
Lateralisation of memory functions in epileptic patients by use of the sodium amytal (Wada) technique
}

\author{
G E POWELL,* C E POLKEY, $\dagger$ A G M CANAVAN $\ddagger$
}

From the Department of Psychology, University of Surrey, ${ }^{*}$ Maudsley Hospital Neurosurgical Unit, $\dagger$ London, Department of Psychology, Institute of Psychiatry, London, $\ddagger$ UK and Psychologisches Institut, Universität Tübingen, $\ddagger$ Tübingen, Federal Republic of Germany

SUMMARY Lateralisation of speech and memory functions was determined in 27 patients, being assessed for surgical treatment of unilateral temporal lobe epilepsy, using the sodium amytal procedure. The most common outcome with regard to language was the retention of normal left hemisphere dominance, regardless of handedness or side of lesion. In contrast, memory functions were most often to be found only in the unaffected hemisphere, also regardless of handedness or side of lesion. A lesion of either hemisphere appeared capable of interfering with language and verbal memory functions in an unexpectedly high proportion of patients.

Lateralisation of cerebral speech dominance, by testing language functions after the intracarotid injection of sodium amytal, was first introduced by Wada in $1949,{ }^{1}$ and has been widely documented since (for example refs 2,3 ). This procedure is usually carried out on patients being assessed for surgical treatment of chronic epilepsy, and plays a major part in determining their suitability for such surgery. We present here data regarding lateralisation of memory functions using a similar technique.

\section{Method}

\section{Subjects}

In all, between 3 February 1976 and 15 January 1986, 46 of the 92 patients being assessed for surgical treatment of chronic epilepsy underwent the intracarotid sodium amytal test. In 19 cases, the procedure was used only to determine side of language or to investigate EEG activity in the two hemispheres separately, and no memory testing was carried out. The remaining 27 patients were all administered memory tests, and it is the results from these patients that are presented here.

Average age at the time of testing was $\mathbf{2 5 . 6}$ years (range,

Address for reprint requests: Dr AGM Canavan, Psychologisches Institut, Gartenstrasse 29, D-7400 Tübingen, Fed Rep Germany.

Received 15 August 1986 and in revised form 13 November 1986. Accepted 17 November 1986
14-43 years). Average age of first seizure was 4.9 years (range, 0-28 years) and average age of onset of chronic seizures was $8 \cdot 1$ years (range, 1-28 years). Chronic onset was before the age of 3 years in seven patients, between 3 and 8 years in ten patients, after 8 years in nine patients and unknown in one other. There were 13 males and 14 females, of whom 18 were right-handed, five were left-handed and three were mixed-handed. One other patient had been initially right-handed, but became forced to use the left hand through the development of a right-sided hemiplegia. For present purposes this patient is therefore also regarded as constitutionally right-handed. There was a known left-sided EEG focus in 14 patients, and a right-sided EEG focus in the remaining 13 . In 14 patients the main reason for carrying out the intracarotid sodium amytal procedure was because the neuropsychological test results were in conflict with the known lesion site, (for example verbal intelligence and verbal memory deficits detected in the presence of a right-sided lesion). In six further cases all neuropsychological functions were found to be impaired in the presence of a unilateral lesion, and in two cases no neuropsychological impairment could be demonstrated. Of the remaining five cases, two underwent the procedure mainly because of sinistrality and two because bilateral involvement was suspected. The medical history in the fifth case was unavailable at the time of the present study. As a result of the intracarotid sodium amytal procedure, 16 patients proceeded to surgery and 11 were refused.

\section{Procedures}

(1) Intracarotid sodium amytal administration

This test is best conducted without any general sedation. 
Local anaesthesia is used in the groin for the introduction of the angiography catheter. Realistically this means that the test can only be used in co-operative teenage or adult patients. The patient has a standard set of scalp EEG electrodes placed and is then brought to the angiogram room, where a suitably experienced radiologist places an angiographic catheter, (Mani type, size FG 5), usually into the right femoral artery. The catheter is then threaded up to one or other internal carotid artery, with appropriate screening and then a small bolus of radiographic contrast medium, (Conray 300) is injected and AP films taken to check the distribution of the medium, which is usually into the anterior and middle cerebral artery territories of that side. The radiologist also measures the dead space in the catheter. A solution of sodium amytal is then prepared by dissolving $250 \mathrm{mg}$ in $10 \mathrm{ml}$ of sterile water. The radiologist is given this solution and he then fills the dead space of the catheter. The patient is asked to hold both arms up, flexed at the elbow, and to make rapid sequential movements of each thumb to the tips of the fingers whilst counting aloud. The radiologist then starts to inject the solution of sodium amytal at a rate of $25 \mathrm{mg}$ every 5 seconds until a hemiparesis of the contralateral arm appears, usually when $75-125 \mathrm{mg}$ have been injected. During this time unilateral EEG changes appearing a few seconds after the injection has started will confirm that mainly one hemisphere has been affected. The psychometric tests as described below are then carried out. This takes about 20 minutes at the end of which time the procedure can either be repeated on the same side or the catheter relocated in the other internal carotid artery. In all 27 cases to be described below the procedure was carried out on both hemispheres. When all the relevant testing has been completed the catheter is removed by the radiologist and appropriate pressure exerted upon the femoral artery to prevent bruising.

\section{(2) Assessment of intellectual functions}

Neuropsychological assessments are carried out routinely as part of the screening procedure for determining suitability for temporal lobe resection. Procedures have varied slightly over the years, but have always included specially adapted short forms of the WAIS ${ }^{4}$ or WAIS-R ${ }^{5}$ and a variety of standardised memory tests, such as the Wechsler memory scale, ${ }^{6}$ recall of the Rey figure ${ }^{7}$ and a visual retention test ${ }^{8}$. Results of such assessments have been described by Powell, Polkey and McMillan. ${ }^{9}$ Patients also receive postoperative reassessments 1 to 6 months after surgery. Details of the present cases (IQ scores and summaries of memory functions) are presented in tables 4,5 and 6 , and postoperative reassessments (where appropriate) are also described in the results section. It should be noted that the short forms of the WAIS or WAIS-R (based upon the comprehension, vocabulary, similarities, block design and object assembly subtests) yield verbal, spatial or performance IQ scores derived from Factor Analysis or Principal Components Analysis which are somewhat more sensitive to various kinds of intellectual impairment than are standard Wechsler IQs (see refs 4 and 5 for details).

Assessment of language and memory functions during the course of the intracarotid sodium amytal test has also varied a little over the years, but the core procedure was as follows. Before administration of the sodium amytal to either hemi- sphere, the patient is taught a simple sentence, such as "Tom's dog ran down the road with a bone in its mouth". This sentence is to be reproduced by the patient upon recovery, and serves as a test of consolidation of the memory trace, since sodium amytal is injected immediately upon memorisation, (the criterion being three successful repetitions). Immediately upon onset of hemiparesis, the patient is questioned as to name and address, serving as a test of both language and memory functions. The patient is then shown a series of three to five line-drawings, for naming aloud. Prior instructions require the patient to try to memorise the material even if naming is not possible, which is of course the case when the hemisphere subserving language is anaesthetised. The procedure continues with the presentation of three to five boldly printed words, for reading aloud and/or memorisation, and then three to eight household objects, for naming and/or memorisation. If hemiparesis is still evident, further ad hoc testing continues (for example simple digit-span or arithmetical tasks) but more often than not the patient is showing signs of mild recovery by this time and so testing is discontinued. Thus either verbal or spatial memory functions may be employed by the patient during this procedure, and so both may contribute to performance in subsequent tests of recall and recognition.

Upon full recovery, usually by about $10 \mathrm{~min}$ following sodium amytal injection, the patient is asked for free recall of any pictures, words or objects presented previously. Ao recognition phase then ensues, wherein the patient is shown again the previous material with two to four novel items randomly interspersed for each kind of material. This allows for the detection of false positive recognition responses, as well as false negatives, and the occurrence of the former casts doubt upon the validity of true positive responses.

Finally the patient is asked for free recall of the sentence learned prior to injection. In all, the procedure takes approximately 20 minutes from beginning to end, and is identical for each injection, with the exception of course of the use of different materials in each trial. The exact materials used have varied somewhat over the years, and have sometimes been adapted to the intellectual level of the patient, but for the most part they have been kept as simple as possible. For instance, the household objects have typically been common items such as a saucer, a pen, scissors or a toy cat. Most patients have been tested with the longest strings of items described above, the shorter strings being employed only with the earliest cases or with repeated testing of one hemisphere.

\section{Results}

There is considerable variability between individuals in the absolute level of free recall or forced choice recognition under the conditions outlined above, and therefore clinical decisions have usually been based upon the relative performances of the two hemispheres. Free recall, for instance, might vary from "zero" to "perfect" for the better hemisphere in different patients. Localisation of memory functions, 
in the former case, would then be deduced from recognition performance alone.

As would be expected, recognition performance is usually superior to free recall, but even here in some cases the difference between the more efficient hemisphere and the lesser might be in the region of five correct recognitions (out of nine to 18 items in total) with no false positives versus one correct recognition with one false positive. In this case the former hemisphere would be regarded as subserving most memory function, with the latter subserving little or none. If less than about three correct recognitions were obtained from either hemisphere it would be concluded that neither hemisphere alone was capable of subserving any useful memory function, (see footnote 1).

In other cases both hemispheres might be shown to contain considerable memory function, both in recognition and recall, with one marginally superior. Finally, the production of a single correct recognition or recall response by a particular hemisphere would not be regarded as evidence of intact memory functioning when that item had been presented late in the procedure, near to the point of recovery. Similarly, as mentioned above, any memory trials including false positive responses were regarded as unreliable and not taken as evidence of intact memory functioning. The results to be reported below, then, are derived mainly from relative rather than absolute values for the two hemispheres. Nevertheless, absolute recall and recognition scores for each patient (where available) are presented in tables $1-3$.

Table 1 Right-handed patients with right-sided lesions

\begin{tabular}{llllll}
\hline & \multicolumn{2}{l}{ Right hemisphere } & & \multicolumn{2}{c}{ Left hemisphere } \\
\cline { 2 - 3 } \cline { 5 - 6 } Patient & Recall & Recognition & & Recall & Recognition \\
\hline PW & 0 & $1^{*}$ & 3 & 3 \\
KS & 0 & FP & & 7 & 15 \\
AD & 0 & 0 & 4 & 15 \\
BR & 0 & $1^{*}$ & & 6 \\
AP & 0 & FP & & 15 \\
TT & 4 & - & 12 & - \\
FJ & 3 & - & 6 & - \\
DM & 0 & 14 & 0 & FP \\
SM & 3 & 10 & 0 & FP \\
BG & 0 & FP & 1 & FP \\
\hline
\end{tabular}

Number of correct responses by each hemisphere in the recall and recognition phases of the intracarotid amytal procedure. First 5 patients, memory functions exclusively in the left hemisphere; next 2 , left hemisphere superior for memory; next 2 , memory functions exclusively in the right hemisphere; final patient, memory functions dependent upon bilateral functioning. Scores were regarded as unreliable when accompanied by False Positive (FP) responses, or when single items were remembered from late in the series $\left({ }^{*}\right)$. Scores for the recognition phase were not available in cases TT and FJ.
Table 2 Right-handed patients with left-sided lesions

\begin{tabular}{llllll}
\hline & \multicolumn{2}{l}{ Right hemisphere } & & \multicolumn{2}{l}{ Left hemisphere } \\
\cline { 2 - 3 } Patient & Recall & Recognition & & Recall & Recognition \\
\hline NI & 1 & 7 & & 0 & 0 \\
AL & 0 & 11 & & $1^{*}$ & FP \\
PT & 8 & - & 0 & - \\
TB & 0 & 14 & & 0 & 16 \\
JN & some & -16 & & some & - \\
AM & 1 & 16 & 9 & - \\
PS & 0 & 0 & 0 & 8 \\
JK & 0 & 0 & 0 & 0 \\
DP & 0 & 3 & FP & 2 \\
\hline
\end{tabular}

Number of correct responses by each hemisphere in the recall and recognition phases of the intracarotid amytal procedure. First 3, memory functions exclusively in the right hemisphere; next 3 , memory functions equally divided between the 2 hemispheres; next 1 , memory functions exclusively in the left hemisphere; final 2, memory functions dependent upon bilateral functioning. Scores were regarded as unreliable when accompanied by False Positive (FP) responses, or when single items were remembered from late in the series $\left.{ }^{*}\right)$. Only a qualitative report was available for patient $\mathrm{JN}$.

Table 3 Left-handed and mixed handed patients

\begin{tabular}{llllll}
\hline & \multicolumn{2}{l}{ Right hemisphere } & & \multicolumn{2}{l}{ Left hemisphere } \\
\cline { 2 - 3 } \cline { 5 - 6 } Patient & Recall & Recognition & & Recall & Recognition \\
\hline BW & 0 & - & 0 & - \\
SW & 1 & 1 & 1 & 3 \\
CH & 1 & 11 & & 0 & FP \\
WC & - & 12 & & - & FP \\
KR & 1 & 7 & & 2 & 15 \\
CC & $1^{*}$ & $1^{*}$ & & 2 & 11 \\
BB & - & good & & FP \\
MB & 1 & 3 & 1 & FP \\
\hline
\end{tabular}

Left-handed patients with right-sided lessions (first 2), or left-sided lesions (next 3) and mixed-handed patients with right-sided lesions (next 1) or left-sided lesions (last 2). Number of correct responses by each hemisphere in the recall and recognition phases of the intracarotid amytal procedure. Scores were regarded as unreliable when accompanied by False Positive (FP) responses, or when single items were remembered from late in the series $\left({ }^{*}\right)$. Only a qualitative report was available for patient BB. See text for interpretation.

\section{(1) Right-handed patients with right-sided lesions, $(n=10)$}

In terms of language, seven patients displayed normal dominance (left hemisphere dominant, with little or no expressive language in the right hemisphere) while three displayed mixed dominance (each hemisphere being able to talk).

With regard to memory, in five patients this was shown to be exclusively in the left hemisphere; in two

Footnote 1. The rationale underlying this decision was derived from the finding (in ten of the earliest cases) that recognition memory for similar materials over a similar length of time was near-perfect when both hemispheres remained functioning throughout. This control procedure was discontinued with later patients because of interference effects between testing sessions. 
Table 4 Right-handed patients with right-sided lesions

\begin{tabular}{|c|c|c|c|c|c|c|c|c|}
\hline \multirow[b]{2}{*}{ Patient } & \multirow[b]{2}{*}{ Sex } & \multicolumn{7}{|l|}{ Onset } \\
\hline & & lst & Chronic & Duration & $V I Q$ & $S I Q / P I Q$ & $V M$ & $S M$ \\
\hline $\begin{array}{l}\text { PW } \\
\text { KS } \\
\text { AD } \\
\text { BR } \\
\text { AP }\end{array}$ & $\begin{array}{l}\mathbf{M} \\
\mathbf{F} \\
\mathbf{F} \\
\mathbf{M} \\
\mathbf{M}\end{array}$ & $\begin{array}{r}9 \mathrm{~m} \\
11 \mathrm{~m} \\
9 \mathrm{~m} \\
4 \mathrm{y} \\
0 \mathrm{y}\end{array}$ & $\begin{array}{l}7 y \\
2 y \\
5 y \\
4 y \\
2 y\end{array}$ & $\begin{array}{l}16 y \\
19 y \\
15 y \\
19 y \\
16 y\end{array}$ & $\begin{array}{r}98 \\
120 \\
78 \\
74 \\
115\end{array}$ & $\begin{array}{l}102 \\
110 \\
121 \\
130 \\
123\end{array}$ & $\begin{array}{l}\text { Good } \\
\text { Good } \\
\text { Good } \\
\text { Impaired } \\
\text { Impaired }\end{array}$ & $\begin{array}{l}\text { Average } \\
\text { Good } \\
\text { Average } \\
\text { Average } \\
\text { Average }\end{array}$ \\
\hline $\begin{array}{l}\text { TT } \\
\text { FJ }\end{array}$ & $\begin{array}{l}\mathbf{M} \\
\mathbf{F}\end{array}$ & $\begin{array}{l}4 \mathrm{y} \\
2 \mathrm{y}\end{array}$ & $\begin{array}{r}15 y \\
4 y\end{array}$ & $\begin{array}{r}5 y \\
11 y\end{array}$ & $\begin{array}{l}89 \\
95\end{array}$ & $\begin{array}{r}113 \\
81\end{array}$ & $\begin{array}{l}\text { Impaired } \\
\text { Good }\end{array}$ & $\begin{array}{l}\text { Good } \\
\text { Average }\end{array}$ \\
\hline $\begin{array}{l}\text { DM } \\
\text { SM }\end{array}$ & $\begin{array}{l}\mathbf{M} \\
\mathbf{M}\end{array}$ & $\begin{array}{l}2 y \\
1 y\end{array}$ & $\begin{array}{l}2 y \\
1 y\end{array}$ & $\begin{array}{l}16 y \\
24 y\end{array}$ & $\begin{array}{l}80 \\
81\end{array}$ & $\begin{array}{l}81 \\
70\end{array}$ & $\begin{array}{l}\text { Impaired } \\
\text { Impaired }\end{array}$ & $\begin{array}{l}\text { Good } \\
\text { Impaired }\end{array}$ \\
\hline BG & $\mathbf{F}$ & $13 y$ & $13 y$ & $22 y$ & 95 & 104 & Average & Good \\
\hline
\end{tabular}

First 5 patients, memory functions exclusively in the left hemisphere; next 2 , left hemisphere superior for memory; next 2 , memory functions exclusively in the right hemisphere; final patient, memory functions dependent upon bilateral functioning. (ONSET refers to age at first epileptic seizure, and age since seizures became chronic. Duration refers to duration of chronic seizures. VIQ is verbal IQ, SIQ/PIQ is Spatial IQ/performance IQ. VM refers to verbal memory functions and SM to spatial memory).

the left hemisphere displayed superior memory but the right hemisphere retained some ability; in two others the right hemisphere displayed superior memory (despite the presence of a lesion in this hemisphere), with the left hemisphere contributing little to this function; and in the other patient neither hemisphere alone subserved any useful memory function. Details of these patients are presented in this same order in tables 1 and 4.

Thus in the first five patients the left hemisphere was seen to be serving all language and memory functions. The first thing to note about these patients is that despite this none of them displayed any deficits in either spatial skills or spatial memory. On the contrary two displayed defects in verbal IQ and two in verbal memory, while two displayed no impairments at all. Neither age of seizure onset nor duration of chronic seizures appear to differentiate these different outcomes.

Since the right hemisphere was not making a significant contribution to either language or memory in these patients, right temporal lobectomy for the relief of seizures was indicated, and so four patients proceeded to surgery (see footnote 2 ), and the postoperative neuropsychological assessments confirmed the above conclusions. PW's VIQ rose to 118 , with no change in any other measures, KS displayed improvements in all memory functions, with no change in IQs, and neither AD nor AP showed any change in IQs but both made considerable gains in memory tests.

In the next two patients in table 4 the left hemisphere displayed superior memory but the right hemisphere retained some ability in this area. Both patients were also mixed dominant for language. One patient (FJ) displayed marginally lower spatial abilities than verbal abilities, but the other displayed the reverse pattern. Neither of these patients had suffered chronic seizures for as long as any of the first five, and both of them had experienced their first seizure at a later age than four of the five. Both proceeded to surgery, after which FJ displayed no changes on any neuropsychological measures while TT displayed improvements on verbal memory tests and in SIQ, with no change in VIQ.

The next two patients in table 4 each displayed superior memory functions in the right (damaged) hemisphere, with the left contributing little to® memory. SM was mixed dominant for language but $\vec{C}$ DM displayed normal language dominance. Both pa- $\triangle$ tients were male and had been suffering with chronico seizures since their very first seizure at an early age. Both patients also displayed fairly generalized mild to moderate impairments in cognitive function. Surgery was contraindicated in both cases.

The final patient in table 4, BG, was of normal language dominance, but neither hemisphere alone was capable of serving any useful memory function, the implication being that the patient needed both hemispheres working in collaboration in order to remember things. Surgery was therefore contraindicated. This patient suffered a late onset epilepsy of many years duration. While she did not display any

Footnote 2. The fifth case, BR, provides an interesting example of the changing role of neuropsychological assessment over the years. BR was one of the first in the present series, and in those days his poor VIQ and verbal memory were taken as evidence of left hemisphere dysfunction. The discovery of right hemisphere dysfunction during the sodium amytal test therefore led to the conclusion that the patient was bilaterally affected, and so surgery was contraindicated. Later in the series, AD presented with a similar picture, but by this time neuropsychological evidence was no longer taken to indicate site of damage, and consequently she proceeded to surgery, with favourable outcome. 
obvious cognitive defects, spatial functions were better preserved than their verbal counterparts.

\section{(2) Right-handed patients with left-sided lesions, $(n=9)$}

In terms of language, five patients displayed normal dominance, two displayed crossed dominance and two displayed mixed dominance, with the left hemisphere nevertheless retaining superiority.

With regard to memory, in three patients this was shown to be exclusively in the right hemisphere; in three patients the two hemispheres were equally good; in one patient the left hemisphere was shown to be superior for memory (despite the presence of a lesion in this hemisphere) with the right hemisphere contributing little to this function; and in the other two patients neither hemisphere alone subserved any useful memory function. Details of these patients are presented in this same order in tables 2 and 5.

In the first two patients the right hemisphere was seen to be serving all language and memory functions, but in the third, PT, although memory was exclusively in the right hemisphere language was shared between both. All three patients were female and the least affected was the one with the earliest known seizure and bilaterally represented language. In the first two cases both verbal and spatial memory were impaired, and in all three verbal intelligence was poorer than spatial intelligence.

NI and PT proceeded to surgery, but EEG recordings at the time of the sodium amytal test demonstrated bilateral disturbance in AL's case and so surgery was contraindicated. NI was suffering from a progressive encephalitis affecting the left hemisphere, and so underwent a subtotal hemispherectomy. Postoperative neuropsychological reassessment revealed improvements in both VIQ (now 62) and PIQ (now 82 ), with delayed verbal memory increasing to $100 \%$ (from $29 \%$ ) although immediate verbal memory was still impaired. Spatial memory rose to a little below average. PT underwent the standard left temporal lobectomy, and postoperatively displayed a VIQ of 81 and an SIQ of 117, with little change in memory functions.

In the next three patients in table 5 each hemisphere made equal contributions to memory function, despite the presence of a left hemisphere lesion. TB and $\mathrm{JN}$ were of normal language dominance, but AM, the least affected of these three males was of mixed language dominance. Verbal IQ was mildly impaired in both TB and JN compared to SIQ. Since the right hemisphere was able to sustain memory functions all three proceeded to surgery. TB showed an increase in SIQ to 146, but neither he nor the other two displayed any change in other IQ scores. Both TB and AM displayed gains in memory functions, TB improving almost to average level, but JN displayed some loss, MQ falling from 74 to 63 .

In the next patient in table 5, PS, the left hemisphere was shown to be of superior memory (despite the presence of a lesion in this hemisphere) with the right contributing little to this function. This patient was also left hemisphere dominant for language. He displayed almost no deficits on any neuropsychological measure, the lower PIQ resulting from poor performance on only one subtest, object assembly. His only other impairment was a considerable left-right disorientation on a streetplan test. In the recall and recognition phase of the sodium amytal test, PS insisted that correctly recognised household objects had been presented to him as words, and that he had not seen them in actuality. Since the left hemisphere was clearly subserving most intellectual functions in this patient despite the presence of an epileptic focus, left temporal lobectomy was contraindicated.

The final two patients in table 5 were each dependent upon the simultaneous functioning of both hemispheres for reliable memory performance. Both

Table 5 Right-handed patients with left-sided lesions

\begin{tabular}{|c|c|c|c|c|c|c|c|c|}
\hline \multirow[b]{2}{*}{ Patient } & \multirow[b]{2}{*}{ Sex } & \multicolumn{7}{|l|}{ Onset } \\
\hline & & lst & Chronic & Duration & $V I Q$ & $S I Q / P I Q$ & $V M$ & $S M$ \\
\hline $\begin{array}{l}\text { NI } \\
\text { AL } \\
\text { PT }\end{array}$ & $\begin{array}{l}\mathbf{F} \\
\mathbf{F} \\
\mathbf{F}\end{array}$ & $\begin{array}{r}12 \mathrm{y} \\
2 \mathrm{y} \\
6 \mathrm{~m}\end{array}$ & $\begin{array}{r}12 y \\
2 y \\
14 y\end{array}$ & $\begin{array}{r}2 y \\
25 y \\
17 y\end{array}$ & $\begin{array}{l}56 \\
81 \\
77\end{array}$ & $\begin{array}{l}62 \\
87 \\
92\end{array}$ & $\begin{array}{l}\text { Impaired } \\
\text { Impaired } \\
\text { Average }\end{array}$ & $\begin{array}{l}\text { Impaired } \\
\text { Impaired } \\
\text { Average }\end{array}$ \\
\hline $\begin{array}{l}\text { TB } \\
\text { JN } \\
\text { AM }\end{array}$ & $\begin{array}{l}\mathbf{M} \\
\mathbf{M} \\
\mathbf{M}\end{array}$ & $\begin{array}{l}8 \mathrm{y} \\
?{ }^{8} \mathrm{~m}\end{array}$ & $\begin{array}{l}8 y \\
7 y\end{array}$ & $\begin{array}{l}11 \mathrm{y} \\
? \\
15 \mathrm{y}\end{array}$ & $\begin{array}{r}81 \\
82 \\
120\end{array}$ & $\begin{array}{l}116 \\
100 \\
119\end{array}$ & $\begin{array}{l}\text { Impaired } \\
\text { Impaired } \\
\text { Average }\end{array}$ & $\begin{array}{l}\text { Impaired } \\
\text { Impaired } \\
\text { Average }\end{array}$ \\
\hline PS & $\mathbf{M}$ & $15 y$ & $15 y$ & $12 y$ & 108 & 90 & Good & Good \\
\hline $\begin{array}{l}\text { JK } \\
\text { DP }\end{array}$ & $\begin{array}{l}F \\
F\end{array}$ & $\begin{array}{l}10 \mathrm{~m} \\
28 \mathrm{y}\end{array}$ & $\begin{array}{r}5 y \\
28 \mathrm{y}\end{array}$ & $\begin{array}{l}22 y \\
15 y\end{array}$ & $\begin{array}{l}106 \\
107\end{array}$ & $\begin{array}{l}84 \\
92\end{array}$ & $\begin{array}{l}\text { Good } \\
\text { Good }\end{array}$ & $\begin{array}{l}\text { Average } \\
\text { Impaired }\end{array}$ \\
\hline
\end{tabular}

First 3 patients, memory functions exclusively in the right hemisphere; next 3 memory functions equally divided between the 2 hemispheres; next 1 , memory functions exclusively in the left hemisphere; final 2 , memory functions dependent upon bilateral functioning. (ONSET refers to age at first epileptic seizure, and age since seizures became chronic. Duration refers to duration of chronic seizures. VIQ is verbal IQ, SIQ/PIQ is spatial IQ/performance IQ. VM refers to verbal memory functions and SM to spatial memory. 
were female and left hemisphere dominant for language. Both displayed better verbal skills and verbal memory than spatial skills and spatial memory. In one case the first known seizure was when very young, but in the other it was late. DP also presented with a history of bilateral amygdalotomy operations, which had failed to control her epilepsy. In each case, then, left temporal lobectomy was contraindicated.

(3) Left-handed and mixed-handed patients, $(n=8)$. There were five left-handed patients, two with rightsided lesions and three with left-sided lesions. Of the three mixed-handed patients, one had a right-sided epileptic focus but the other two had left-sided lesions. Details of these patients are presented in this same order in tables 3 and 6.

The first two patients were both left-handed with right-sided lesions. They both displayed normal language dominance. In neither case was either hemisphere alone able to subserve useful memory function. In both cases verbal memory was impaired but spatial memory was good, and in one case spatial skills were superior to verbal skills. Right temporal lobectomy was contraindicated for both.

The next three patients were all left-handed with left-sided lesions. Despite this $\mathrm{CH}$ was of normal language dominance, although memory functions resided exclusively in the right hemisphere. Her only impairment was of spatial memory. She proceeded to left temporal lobectomy, with no effect upon IQ scores, but all memory functions improved to within the normal range. WC was crossed dominant for language, and once again all memory functions were to be found in the right hemisphere. His only impairment was in reduced VIQ compared to PIQ. He also proceeded to left temporal lobectomy, with no effect upon neuropsychological test scores. Finally $\mathrm{KR}$ was also crossed dominant for language, but in her case memory functions were subserved more by the left hemisphere than the right. Verbal skills and verbal memory were superior to spatial skills and spatial memory. The presence of a known tumour in the left temporal lobe in this case encouraged resort to surgery. Postoperative reassessment revealed a loss in VIQ, which fell to 84 , and losses in verbal memory functions, which fell into the impaired range, with no change in PIQ or spatial memory.

The only mixed-handed patient with a right-sided lesion, CC, proved to be of normal dominance for language. The left hemisphere also subserved all memory functions. His only impairment was of PIQ in relation to VIQ. He proceeded to right temporal lobectomy with little change on any neuropsychological measures.

Finally, details of the two mixed-handed patients with left-sided lesions are presented at the bottom of table 6. BB proved to be of mixed dominance for language, but memory functions were subserved exclusively by the right hemisphere. She proceeded to left temporal lobectomy with no effect on IQ scores or spatial memory, but verbal memory improved to just below average. MB was of normal language dominance, despite the presence of a left-sided lesion. Her verbal skills and verbal memory were also superior to her spatial skills and spatial memory. However, memory functions were dependent upon simultaneous functioning of the two hemispheres, with neither hemisphere alone producing reliable memory performance. Left temporal lobectomy was therefore contraindicated.

Tables 7 and 8 present summary findings regarding localisation of language and memory functions respectively.

\section{Discussion}

Over a ten year period 92 patients were assessed for surgical treatment of chronic temporal lobe epilepsy. Of these, 27 were administered memory tests during the sodium amytal procedure, and in 22 cases this was

Table 6 Left-handed and mixed handed patients

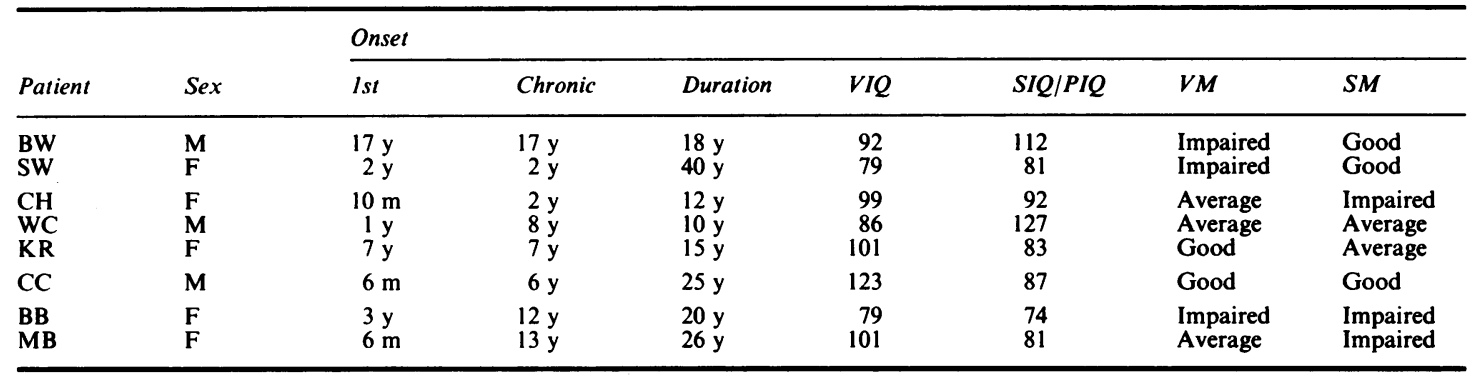

Left-handed patients with right-sided lesions (first 2), or left-sided lesions (next 3) and mixed-handed patients with right-sided lesions (next 1) or left-sided lesions (last 2). (ONSET refers to age at first epileptic seizure, and age since seizures became chronic. Duration refers to duration of chronic seizures. VIQ is verbal IQ, SIQ/PIQ is spatial IQ/performance IQ. VM refers to verbal memory functions and SM to spatial memory). 
Table 7 Language dominance as determined by intracarotid sodium amytal testing in 27 epileptic patients

\begin{tabular}{lllllc}
\hline & $R h / R l$ & $R h / L l$ & $L M h / R l$ & $L M h / L l$ & Total \\
\hline Normal dominance & 7 & 5 & 3 & 2 & 17 \\
Crossed dominance & 3 & 2 & & 2 & 4 \\
Mixed dominance & 2 & 1 & 6 & \\
\hline
\end{tabular}

$\mathbf{R h}=$ right-handed; $\mathbf{L M h}=$ left- or mixed-handed; $\mathbf{R l}=$ right lesion; $\mathbf{L l}=$ left lesion.

Table 8 Location of memory functions as determined by intracarotid sodium amytal testing in 27 epileptic patients

\begin{tabular}{|c|c|c|c|c|c|}
\hline & $R h / R l$ & $R h / L l$ & $L M h / R l$ & $L M h / L i$ & Total \\
\hline $\begin{array}{l}\text { Normal (bilateral) } \\
\text { Exclusively unilateral, opposite lesion } \\
\text { Mostly unilateral, opposite lesion } \\
\text { Exclusively unilateral, with lesion } \\
\text { Mostly unilateral, with lesion } \\
\text { Neither alone }\end{array}$ & $\begin{array}{l}5 \\
2 \\
2 \\
1\end{array}$ & $\begin{array}{l}3 \\
3 \\
1 \\
2\end{array}$ & 1 & $\begin{array}{l}3 \\
1 \\
1\end{array}$ & $\begin{array}{r}3 \\
12 \\
2 \\
3 \\
1 \\
6\end{array}$ \\
\hline
\end{tabular}

$\mathrm{Rh}=$ right-handed; $\mathbf{L M h}=$ left- or mixed-handed; $\mathbf{R I}=$ right lesion; $\mathrm{Ll}=$ left lesion.

because neuropsychological results were inconsistent with the known lesion site. Slightly less than a quarter of all referrals, then, displayed unexpected patterns of intellectual function in response to temporal lobe epilepsy. For the most part, data gathered during the sodium amytal procedure confirmed this picture.

Considering the fate of language functions first, it is clear from table 7 that the commonest outcome of focal temporal lobe epilepsy, regardless of handedness or side of lesion, is the retention of normal left hemisphere language dominance, as displayed by $63 \%$ of patients in the present study. Mixed language dominance is seen in a further $22 \%$, mostly in righthanded patients and once again regardless of side of lesion. The least common outcome is crossed dominance for language (15\%) seen only after left-sided lesions and regardless of handedness.

While it is difficult to oust language from its preferred hemisphere $(85 \%$ of patients therefore displayed at least as good language in the left as in the right) it is not so difficult to produce impairment of this function. Furthermore a lesion in either hemisphere can have this effect. Considering only the righthanded patients, then five of the nine with left-sided lesions displayed reduced VIQ as compared to SIQ/PIQ (see table 5), as did seven of the ten with right-sided lesions (see table 4). There was no obvious relationship between location of language functions and presence of language impairment.

Turning now to memory functions, a very different picture emerges. In the normal person memory would be expected to be bilaterally organised. In the present sample only $11 \%$ displayed such normality (see table 8) and these were all right-handed patients with left-sided lesions. The commonest outcome was for memory to be found exclusively unilateral, opposite the side of the lesion $(44 \%)$, and regardless of hand- edness or side of lesion. This figure could be supplemented by a further $7 \%$ in whom memory was found to be mostly unilateral and opposite the lesion.

In $22 \%$ of patients memory could not be demonstrated convincingly in either hemisphere alone. This again was regardless of handedness or side of lesion. Thus, while in a sense it could be claimed that memory was bilaterally organised in these patients, it certainly could not be claimed that such organisation was normal. A further $15 \%$ of patients displayed a curious exclusive or mostly unilateral location of memory functions within the hemisphere known to be responsible for epileptic seizures. This would suggest undetected lesions in the other hemisphere, making that hemisphere even more inhospitable toward memory functions than the epileptic one, a suggestion which cannot be confirmed during life.

As regards disruption of function (rather than location of function) it would appear that verbal memory is far more susceptible to the effects of unilateral temporal lobe epilepsy of either hemisphere than is spatial memory. In this respect the results resemble those for language functions. Considering only the right-handed patients, verbal memory was disrupted in four of the nine with left-sided lesions (table 5) but also five of the ten with right-sided lesions (table 4). In contrast, and surprisingly, spatial memory was affected in only one of the ten with rightsided lesions but five of the nine with left-sided lesions. In general, in right-handers, left-sided lesions produced little discrepancy between verbal memory and spatial memory, while right-sided lesions produced marked discrepancies, in either direction. As before there was no obvious relationship between location of memory functions and presence of memory impairment.

The conclusion that verbal memory is more sus- 
ceptible to the effects of unilateral lesions than is spatial memory does however warrant qualification. It might be for instance that assessment techniques for quantifying verbal memory are simply more sensitive than those available for the assessment of spatial memory. This possibility is unlikely though, since it would lead to a higher detection rate for verbal memory problems than for spatial memory deficits not just under the present conditions but also in the assessment of temporal lobe epileptics in general, and there is no evidence that this is the case. ${ }^{49}$ Similarly, it might be that it is easier to compensate for spatial memory defects by utilising verbal strategies than it is to compensate for verbal memory defects by utilising spatial strategies. Once again, though, this should lead to a higher incidence of verbal memory deficits in general, and not just in those patients selected for the sodium amytal procedure. Also, were it accepted that spatial memory deficits are easily compensated, then it would beg the question as to why such deficits do indeed persist in many patients with many different types of lesion (e.g. ref 10).

It was remarked earlier that individual performance during the intracarotid sodium amytal test was highly variable, and that results were often based upon relative performances of the two hemispheres rather than absolute scores. Hence between-subjects comparisons have not been presented here. The certainty of the results would therefore be highly questionable were it not for the outcomes following surgery presented here. Without exception all patients showed changes in function consistent with predictions derived from the intracarotid sodium amytal test, mostly for the better but unfortunately in one case for the worse. All such patients have also shown a marked reduction in seizure frequency.

Finally, while the results presented here are instructive with regard both to the location and to the level of functioning of memory in epileptic patients, they do not throw any light upon mechanisms of memory. For the most part the materials used in the sodium amytal procedure may have been encoded in either verbal or spatial memory, and there is no certain way of determining which of these forms of memory was actually employed by the patient while anaesthetised. It is likely, however, that each patient relied upon whichever form was the more intact in that particular individual. The most interesting observation in this respect was made on the patient PS, in whom the left hemisphere was seen to be subserving all memory functions. In recognition tests this patient insisted that household objects had not been presented to him as real objects but rather as written words. This comment was made spontaneously, and to date no systematic attempt to study the subjective experiences of patients undergoing the intracarotid amytal procedure has been made.

In summary the data presented here are characterised by great variability between subjects, with no obvious relationships to handedness, side of lesion, onset of seizures or duration of seizures. It may be of course, that such relationships exist, and would reveal themselves with a much larger sample of patients, but at the current rate of 2.7 per year these data are a long way into the future. A number of consistencies, however, have emerged. First, language is much less likely to show displacement of location than memory. Second, both language functions and verbal memory may be impaired by unilateral epilepsy of either hemisphere, and regardless of either handedness or actual location of these abilities in the brain. Finally, in righthanded patients, left-sided lesions leave verbal and spatial abilities relatively symmetrical (though at many different absolute levels) while right-sided lesions tend to produce verbal-spatial discrepancies, in either direction. These results are not entirely consistent with the classical views of clinical neuropsychology.

\section{References}

1 Wada JA. A new method for the determination of the side of cerebral speech dominance. A preliminary report on the intracarotid injection of sodium amytal in man. Igaku to Siebutsugaku 1949;14:221-2.

2 Wada JA, Rasmussen T. Intracarotid injection of sodium amytal for the lateralization of cerebral speech dominance. $J$ Neurosurg 1960;17:266-82.

3 Rausch R, Fedio P, Ary CM, Engel J, Crandall PH. Resumption of behaviour following intracarotid sodium amobarbital injection. Ann Neurol 1984;15:31-5.

4 Powell GE. The relationship between intelligence and verbal and spatial memory. $J$ Clin Psychol 1979;35:335-40.

5 Canavan AGM, Dunn G, McMillan TM. Principal components of the WAIS-R. Br J Clin Psychol 1986;25:81-5.

6 Wechsler D, Stone CP. Wechsler Memory Scale. Manual. New York, Psychological Corporation 1945.

7 Teuber HL, Milner B, Vaughan HG. Persistent anterograde amnesia after stab wound of the basal brain. Neuropsychologia 1968;64:267-82.

8 Benton AL. The Revised Visual Retention Test. New York, Psychological Corporation 1955.

9 Powell GE, Polkey CE, McMillan T. The new Maudsley series of temporal lobectomy. I: Short-term cognitive effects. Br J Clin Psychol 1985;24:109-24.

10 Kolb B, Whishaw IQ. Fundamentals of Human Neuropsychology. San Francisco, WH Freeman 1980. 